\title{
A Platform for High Performance and Flexible Virtual Routers on Commodity Hardware
}

\author{
Norbert Egi, Mickael Hoerdt, Laurent Mathy, Panagiotis Papadimitriou \\ Computing Dept., Lancaster University, UK \\ \{n.egi, m.hoerdt, I.mathy, p.papadimitriou\} @ lancaster.ac.uk \\ Adam Greenhalgh, Mark Handley \\ Dept. of Computer Science, University College London, UK \\ \{a.greenhalgh, m.handley\}@cs.ucl.ac.uk \\ Felipe Huici \\ NEC Europe Ltd, Germany \\ felipe.huici@nw.neclab.eu
}

\begin{abstract}
Multi-core CPUs, along with recent advances in memory and buses, render commodity hardware a strong candidate for software router virtualization. In this context, we present the design of a new platform for virtual routers on modern PC hardware. We further discuss our design choices in order to achieve both high performance and flexibility for packet processing.
\end{abstract}

\section{Categories and Subject Descriptors}

C.2.6 [Computer Communication Networks]: [InternetworkingRouters]

\section{General Terms}

Design

\section{Keywords}

Virtualization, Routers, Commodity Hardware

\section{INTRODUCTION}

Recent research has shown that modern PCs are a viable platform for the implementation of high-performance software routers [1, 2]. The functionality provided by software routers combined with recent virtualization technologies allows multiple instances of routers to run concurrently on a single box while offering highly configurable forwarding planes and custom routing protocols. Some recent PC-based virtual router prototypes [3] have been proposed, but none of them exploit recent advances in commodity hardware such as multi-core CPUs or network interface cards with hardware multi-queuing.

In [1] we showed that the main performance bottleneck for PCbased software routers is main memory access. With the memory subsystem as the limiting factor for at least the next few years, the number of spare CPU cycles is likely to increase. To exploit these resources we can certainly consolidate a set of software routers running concurrently on the same hardware, inline with conventional server virtualization. However, the short-lived nature of packets inside a router makes router virtualization challenging and certainly more demanding. Is it possible to have the best of two worlds, building a flexible virtual router platform that also reaches the performance limits of the underlying hardware?
Motivated by this challenge, we present a new platform for software virtual routers on commodity hardware. The platform leverages modern and emerging hardware trends to provide: (i) consolidation of virtual data planes onto a common forwarding domain, (ii) highly configurable forwarding planes for advanced programmability, and (iii) the forwarding tree ${ }^{1}$ as the basic resource allocation unit for the mapping of virtual router components to cache hierarchies.

\section{PLATFORM DESIGN OVERVIEW}

The design of our virtual router platform is mainly driven by performance and flexibility. We use Xen's [4] paravirtualization to host the guest domains and Click [5] for packet processing and forwarding.

The platform comprises the following basic components (Fig. 1): (i) a management domain for the management of guest domains, (ii) an isolated driver domain (IDD) for aggregated packet forwarding, and (iii) a number of guest domains for hosting control planes (one per virtual router), and optionally forwarding planes when increased isolation and safety properties are required.

The IDD is a virtual machine that has physical devices mapped to it and runs the appropriate device driver and router software required to host our forwarding planes (FPs). The IDD hosts the merged forwarding paths and provides the ability to control and configure the individual FPs to their respective guest domains. The merging process enables the consolidation of all FPs within the IDD, allowing a large number of virtual routers to share common network interfaces. In [1], we showed that forwarding within a common domain provides significantly higher performance than forwarding in the separate guest domains by avoiding costly perpacket hypervisor domain context switches. Fig. 1 depicts this configuration with FP1 running in the IDD.

The platform supports two additional packet forwarding configurations: (i) splitting a FP between an IDD and a separate guest domain while using local I/O channels for inter-domain communication (e.g., FP2 and FP2' in Fig. 1); and (ii) mapping interfaces directly into guest domains so that each FP resides in a separate guest domain (e.g., FP3 in Fig. 1). Configuration (i) can be used to safely run FPs that include untrusted Click elements without compromising the performance and safety of other virtual routers' FPs.

\footnotetext{
${ }^{1} \mathrm{~A}$ forwarding tree is the set of packet processing elements necessary to move a packet from a single input to all possible outputs.
} 


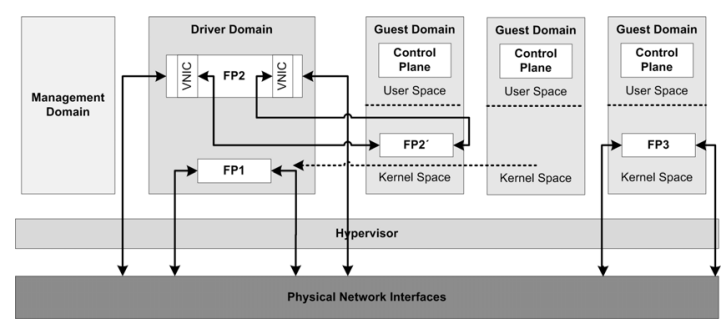

Figure 1: Platform overview.

In configuration (ii) we can directly access the network interfaces from a guest domain, but only by allocating whole (unshared) interfaces to the domain, resulting in coarse network resource sharing. To overcome this issue, we need virtualized network interface support both on the hardware side (e.g., Virtual Machine Devices Queue, VMDq) as well as on the software side (i.e., in the Xen Hypervisor).

The control planes for the virtual routers reside in the guest domains. The platform supports off-the-shelf control plane solutions running in user space, such as XORP or Quagga. For further details on the platform design and implementation, see [6].

\section{FORWARDING PATH ARCHITECTURE}

In order to maximize the performance of PC-based virtual routers, the CPU cores and the cache memory hierarchies need to be carefully exploited. A cache hierarchy is the set of caches within one CPU package, with a PC potentially containing several CPUs and thus several hierarchies. As a result, the aim when implementing a virtual router should be to keep a packet as deep as possible inside a cache hierarchy (i.e., close to the cores) while distributing the packet processing over as many spare cores as possible within the same cache hierarchy. This ensures that processing is not CPUlimited since multiple cores are in use, while reducing expensive accesses to main memory.

A software router's internal organization can be viewed as a graph of interconnected packet processing elements. With this in mind, achieving high performance is non-trivial: while allocating a whole router onto a cache hierarchy is possible, it may not always be desirable, since confining the whole of a software router to only a subset of the cores in the system would result in poor resource utilization.

Even though it is not possible to know in advance to which output interfaces packets have to be switched, they should be kept within a single cache hierarchy whilst distributing the packet processing over as many spare cores as possible within that hierarchy. The key to solving this is to realize that a router's graph organization can be decomposed into a series of forwarding trees. Each of these forwarding trees is associated with an input interface and represents all of the possible forwarding paths followed by packets entering through that interface. Fig. 2 illustrates such a configuration where three forwarding trees are rooted at the input interfaces, each with its own set of independent elements. The advantage of using a forwarding tree is that its elements can all be allocated to the same cache hierarchy, thus confining packets to this hierarchy and reducing main memory accesses. In addition, forwarding trees compose a smaller allocation unit than routers, providing more flexibility when exploiting hardware resources and implementing fairness. In order to avoid conflicts among the trees during the lookup operation, we replicate the forwarding information base on all cache hierarchies.

Besides the concept of forwarding trees, Fig. 2 demonstrates fur-

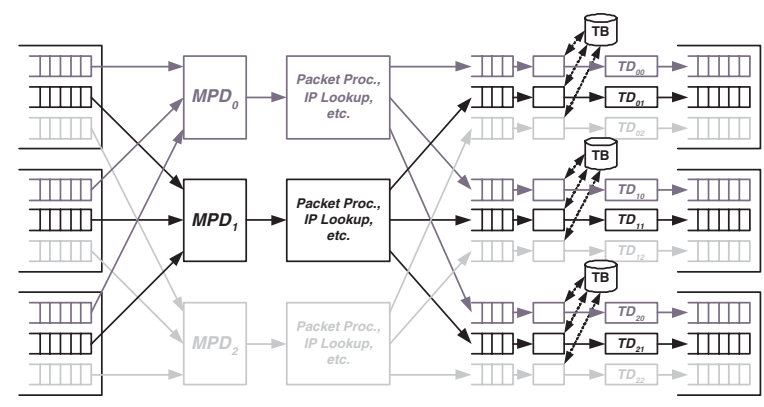

Figure 2: Forwarding path architecture. MPD stands for multiple poll device, TB for token bucket and TD for todevice.

ther properties of our forwarding architecture. First of all, by using hardware multi-queueing and classification based on VMDq available in recent network interface cards (NIC), each interface (both input and output) is split into as many hardware queues as there are virtual routers (VRs). This organization ensures that every interface is shared (i.e. virtualized) among the VRs and every VR can access the interfaces in parallel. Of course, this also ensures that multiple cores can access the interfaces in parallel, providing significantly improved performance. However, being able to access an interface simultaneously still does not prevent the system from the costly overhead of a context-switch when an empty hardware queue is accessed. To mitigate this overhead, we propose to extend Click's original PollDevice element, so that multiple input queues (from multiple input ports) can be assigned to a single element. This reduces the probability that no packets will be processed by an element when it gets scheduled.

Using hardware multi-queueing on the output ports results in improved performance as well, due to the true parallelization given by the independent access to multiple queues. Having said that, it is worth noting that once a packet has been placed in a hardware queue, it is out of the control of the router. As a result, if the NIC does not support certain features such as traffic management or advanced scheduling policies, these must be handled in software by coordinating the output tasks involved. This, in turn, brings back the need for synchronization primitives (e.g., locks) and/or data structures (e.g., token buckets), as illustrated in Fig. 2.

\section{REFERENCES}

[1] N. Egi, A. Greenhalgh, M. Handley, M. Hoerdt, F. Huici, and L. Mathy, "Towards high performance virtual routers on commodity hardware," in Proceedings of ACM CoNEXT 2008, Madrid, Spain, December 2008.

[2] K. Argyraki, S. A. Baset, B.-G. Chun, K. Fall, G. Iannaccone, A. Knies, E. Kohler, M. Manesh, S. Nedveschi, and S. Ratnasamy, "Can software routers scale?" in Proceedings of PRESTO'08, Seattle, USA, August 2008.

[3] S. Bhatia, M. Motiwala, W. Muhlbauer, Y. Mundada, V. Valancius, A. Bavier, N. Feamster, L. Peterson, and J. Rexford, "Trellis: A platform for building flexible, fast virtual networks on commodity hardware," in Proceedings of ACM ROADS '08, Madrid, Spain, December 2008.

[4] P. Barham, B. Dragovic, K. Fraser, S. Hand, T. Harris, A. Ho, R.Neugebauer, I.Pratt, and A. Warfield, "Xen and the art of virtualization," in 19th ACM Symposium on Operating Systems Principles. ACM Press, October 2003.

[5] E. Kohler, R. Morris, B. Chen, J. Jahnotti, and M. F. Kasshoek, "The click modular router," ACM Transaction on Computer Systems, vol. 18, no. 3, pp. 263-297, 2000.

[6] N. Egi, A. Greenhalgh, M. Handley, M. Hoerdt, F. Huici, L. Mathy, and P. Papadimitriou, "The virtual router project," http://nrg.cs.ucl.ac.uk/vrouter/. 